INSTITUT NATIONAL DE LA STATISTIQUE ET DES ETUDES ECONOMIQUES Série des Documents de Travail du CREST (Centre de Recherche en Economie et Statistique)

\title{
$n^{\circ} 2002-10$
}

\section{Salaire minimum et emploi en présence de négociations salariales}

G. LAROQUE

B. SALANIE 2

Les documents de travail ne reflètent pas la position de l'INSEE et n'engagent que leurs auteurs.

Working papers do not reflect the position of INSEE but only the views of the authors.

\footnotetext{
1 CREST-INSEE et CNRS URA 2200.

2 CREST-INSEE, CNRS URA 2200 et CEPR.

Nous remercions Francis Kramarz, Jean-Marc Robin, Jean-Charles Rochet et William Thomson pour leur aide. Les erreurs et imperfections de cet article nous sont entièrement imputables.

Correspondance à Bernard Salanié : CREST-INSEE, 15 Boulevard Gabriel Péri, 92245 Malakoff Cédex, France.

Tél. : 01411760 79. Fax : 01411760 29. Email : salanie@ensae.fr
} 


\title{
Salaire minimum et emploi en présence de négociations salariales
}

\author{
Guy Laroque* $\quad$ Bernard Salanié ${ }^{*}$
}

Janvier 2002

\author{
Correspondance à Bernard Salanié \\ CREST-INSEE \\ 15 bd Gabriel-Péri \\ 92245 Malakoff \\ Tel : 0141176079 \\ Fax : 0141176029 \\ Email : salanie@ensae.fr
}

${ }^{*}$ CREST-INSEE et CNRS URA 2200.

${ }^{\dagger}$ CREST-INSEE, CNRS URA 2200 et CEPR. Nous remercions Francis Kramarz, Jean-Marc Robin, Jean-Charles Rochet et William Thomson pour leur aide. Les erreurs et imperfections de cet article nous sont entièrement imputables. 


\title{
Résumé
}

L'objet de cet article est d'étudier l'effet des variations du salaire minimum sur l'emploi et la distribution des salaires quand ceux-ci sont négociés de manière individuelle entre l'entreprise et le salarié. Une hausse de salaire minimum peut alors augmenter le bien-être des travailleurs si l'augmentation induite des salaires compense les pertes d'emploi. Afin de quantifier ces effets, nous procédons à l'estimation d'un modèle inspiré de nos travaux antérieurs sur données individuelles. Les données rejettent la prédiction du modèle selon laquelle le salaire négocié dépend positivement des variables qui réduisent la participation.

\begin{abstract}
This paper studies the effect of changes in the minimum wage on employment and the wage distribution when wages are bargained over by each employee and each employer. Then an increase in the minimum wage may increase the welfare of workers if the induced wage increase more than compensates for job losses. In order to quantify these effects, we estimate a model inspired by our earlier work on individual data. The data reject the prediction of the model that wages depend positiveley on the variables that reduce participation.
\end{abstract}




\section{Introduction}

L'objet de cet article est d'essayer de préciser les effets des variations du salaire minimum sur l'emploi et la distribution des salaires sous l'hypothèse d'une fixation des salaires par négociation. Nous partons d'un modèle de détermination de l'emploi inspiré de nos travaux antérieurs (Laroque et Salanié (2000, 2001a, 2001b)), qui retient deux principales causes de non-emploi : l'effet sur la participation du système socio-fiscal et l'effet du salaire minimum sur la possibilité d'obtenir un emploi. Ce type de modèle rend bien compte en coupe instantanée des différences observées des taux d'emploi féminins dans la population, selon les revenus du conjoint, le diplôme, le nombre et l'âge des enfants, etc. En variante, lorsqu'on augmente le salaire minimum, nous avons supposé dans nos travaux antérieurs que dans le long terme les employées rattrapées par le salaire minimum perdent leur emploi, alors que le coût du travail d'une employée qui conserve son emploi reste inchangé. Cette hypothèse doit être remise en cause si on pense que les variations du salaire minimum ont des répercussions durables sur la forme de la distribution des salaires versés au dessus du SMIC, par exemple parce qu'elles modifient le pouvoir de négociation des salariés peu qualifiés. La présente étude a pour but d'évaluer l'importance pratique de cette remise en cause.

De nombreux développements récents en économie du travail ont en leur cœur l'idée que les salaires font l'objet d'un marchandage bilatéral entre l'employeur et le salarié. C'est le cas notamment du modèle WS-PS développé à la suite de Layard-Nickell-Jackman (1991), mais aussi des modèles d'appariement (Pissarides (2000)) qu'on présente souvent comme la théorie moderne du chômage d'équilibre. Cependant, la théorie de la négociation salariale sous-jacente n'a fait l'objet que de tests très indirects. Ainsi, Layard-Nickell-Jackman n'examinent cette question que sur des données agrégées; ils présentent des résultats qui suggèrent que le niveau et surtout la durée de versement des allocations chômage ont un effet positif sur le chômage. Beaucoup de travaux empiriques assimilent le pouvoir de négociation des travailleurs (que nous noterons désormais $\alpha$ ) au paramètre de partage des quasi-rentes engendrées par l'activité de l'entreprise. Leurs auteurs ont estimé des régressions qui rapportent le taux de salaire dans une branche donnée à un indicateur de la capacité à payer des entreprises de cette branche. Abowd et Lemieux (1993) instrumentent ainsi la capacité à payer par les prix des importations et des exportations sur données canadiennes et trouvent que le poids des travailleurs dans la négociation est de l'ordre de 0,2. Abowd et Allain (1996) améliorent cette approche en utilisant les estimations des effets-personnes de Abowd, Kramarz et Margolis (1999) pour estimer le salaire de réservation des travailleurs. Ils obtiennent sur données françaises une valeur de $\alpha$ proche de 0,4. Blanchflower, Oswald et Sanfey (1996) utilisent des données portant sur l'industrie américaine et obtiennent une estimation de $\alpha$ plus faible que les précédentes. Van Reenen (1996) obtient une valeur de 0, 3 sur données britanniques et Gianella (2002) et Kramarz (2002) des valeurs proches de 
0,2 sur données françaises. Quant à la littérature économétrique sur les modèles d'appariement, elle suppose le plus souvent que les salaires sont affichés par les entreprises et ne font pas l'objet d'un marchandage. Parmi les exceptions, on peut citer Eckstein et Wolpin (1995) qui retiennent l'idée d'un marchandage individuel entre l'entreprise et chaque salarié, mais ne peuvent identifier le paramètre $\alpha$. Flinn (2001) obtient des estimations de $\alpha$ qui sont assez imprécises, du fait de la difficulté de l'identifier en pratique.

Ces articles reposent généralement sur une spécification très simple du point de menace du travailleur, qui est expliqué par le niveau des allocations chômage, le salaire moyen de la catégorie de travailleurs concernés, et le taux de chômage. Dans la réalité, les travailleurs sont exposés à des taux de prélèvement fiscaux marginaux très variables; par ailleurs, leurs désutilités du travail sont aussi extrêmement hétérogènes. Les points de menace des différents travailleurs varient donc considérablement. Leur distribution dans la population peut être estimée en modélisant la décision de participation des travailleurs. Lorsque l'entreprise ou le syndicat patronal fait face à un syndicat représentant un groupe de travailleurs hétérogènes, la modélisation du processus de négociation collective pose des problèmes difficiles à résoudre. On peut imaginer que le syndicat ait pour objectif une fonction de bien-être social des travailleurs qu'il représente; en théorie, pour être représentatif, le syndicat doit alors disposer d'informations très détaillées sur la situation de chacun de ses adhérents. Par ailleurs, la spécification habituelle du problème de marchandage suppose que l'employeur dispose de cette même information, y compris pour les adhérents au syndicat qui n'obtiennent finalement pas d'emploi. Ces conditions paraissent très fortes.

Il n'existe en fait pas à notre connaissance de modèle théorique simple, aisément confrontable aux données individuelles, de la négociation salariale collective. Faute de mieux, nous avons contourné la difficulté en supposant que la négociation prend place au niveau individuel, entre l'entreprise et chaque salarié potentiel. Ceci implique que le salaire négocié dépend de manière complexe de quatre groupes de variables : celles qui affectent la productivité, celles qui affectent la désutilité du travail, celles qui entrent en jeu du fait du système socio-fiscal, et enfin le salaire minimum. Nous estimons ce modèle sur un échantillon de femmes tiré de l'Enquête Emploi 1999. Comme le salaire minimum est constant dans cet échantillon, le modèle de négociation salariale est identifié à travers ses prédictions sur l'effet du deuxième et du troisième groupe de variables sur les salaires des employées. L'estimation jointe de l'équation de participation et de l'équation de salaires permet l'identification du pouvoir de négociation des travailleurs, conditionnellement à la forme que prend la solution du marchandage.

La section 1 montre que la solution de Nash conduit à des effets du salaire minimum sur la distribution des salaires qui s'ajustent mal sur données françaises; elle explique comment la variante introduite par Kalai-Smorodinsky ne présente pas ces inconvénients. Le reste de l'article retient la solution de KalaiSmorodinsky. La section 2 détaille la spécification du modèle et le calcul de sa 
fonction de vraisemblance. Les résultats d'estimation sont présentés en section 3. Il apparaît que les données rejettent le modèle de négociation salariale au niveau individuel en faveur du modèle concurrentiel. Néanmoins, nous analysons en section 4 l'effet de différentes variables sur le salaire négocié quand celui-ci ne coïncide pas avec la productivité. Enfin, la section 5 étudie l'effet du salaire minimum sur l'emploi, la distribution des salaires et plusieurs mesures du bien-être en fonction du pouvoir de marché de l'employeur.

\section{La théorie de la négociation salariale}

Les théories de la négociation salariale supposent souvent que chaque entreprise (ou syndicat d'employeurs) négocie avec un syndicat de travailleurs représentatif d'un "bassin d'emploi" (voir par exemple le chapitre 5 de Cahuc et Zylberberg (2000)). Le concept de bassin d'emploi est en fait assez flou et il serait difficile avec les données dont nous disposons de tester cette hypothèse qui devrait faire l'objet de travaux ultérieurs. Nous supposons dans cet article que chaque entreprise négocie avec chaque travailleur au niveau individuel, et que cette négociation ne fait pas référence aux salaires versés aux autres travailleurs employés dans l'entreprise. C'est une hypothèse extrême, qui prend en compte la forte hétérogénéité des situations individuelles.

Dans les modèles les plus simples, chaque individu est caractérisé par une productivité $\rho$ et une désutilité du travail $D$. Le passage de la rémunération au revenu disponible met en jeu le système socio-fiscal à travers le barème complexe de prélèvements et de transferts. Nous le résumons par une fonction $R$ qui associe au coût du travail $w$ supporté par l'employeur le revenu net $R(w)$ du ménage de la personne concernée. La fonction $R$ dépend du salaire du conjoint et retrace le jeu des cotisations sociales, de la CSG et de la CRDS, de l'impôt sur le revenu, des prestations familiales, des allocations logement, de la taxe d'habitation et du RMI. Elle dépend de manière implicite mais cruciale des caractéristiques du ménage : présence et salaire éventuel d'un conjoint, nombre et âge des enfants, type de logement... Nous supposerons dans cet exposé théorique que $R$ est continûment différentiable et strictement croissante; nous reviendrons plus loin sur cette hypothèse.

Nous négligeons les possibilités d'emploi à temps partiel. Un emploi à temps plein rémunéré au coût du travail $w$ rapporte $(\rho-w)$ à l'employeur et $(R(w)-D)$ à l'employé; en l'absence d'accord, le travailleur a une utilité donnée par $R(0)$ et l'entreprise un profit nul. Le coût du travail $w$ est compris entre le coût du salaire minimum $\underline{w}$ (la législation est appliquée) et la productivité $\rho$ (sinon l'entreprise préférerait ne pas employer la personne); on en déduit immédiatement que si $\rho<\underline{w}$ ou si $R(\rho)<R(0)+D$, le travailleur sera sans emploi, et ce quel que soit le concept de solution adopté. Supposons maintenant que $\rho>\underline{w}$ et $R(\rho)>R(0)+D$. Alors il existe des valeurs de $w$ telles que l'emploi procure un surplus positif aux 
deux parties. Considérons par exemple la solution de Nash pondérée. Celle-ci affecte un pouvoir de négociation $0<\alpha<1$ au travailleur, et résout le programme

$$
\max _{w \geq \underline{w}}(R(w)-D-R(0))^{\alpha}(\rho-w)^{1-\alpha}
$$

Il est facile de voir que si $R$ est concave (ce qui traduit un barème d'imposition nette progressif sur le domaine considéré), l'objectif est strictement log-concave. Un calcul simple montre que si

$$
\rho<\bar{\rho}=\underline{w}+\frac{1-\alpha}{\alpha} \frac{R(\underline{w})-D-R(0)}{R^{\prime}(\underline{w})}
$$

alors l'objectif est décroissant sur $[\underline{w}, \rho]$ et le salaire négocié est $w=\underline{w}$. Quand $\rho>\bar{\rho}$, le salaire négocié est défini de manière unique par la condition du premier ordre

$$
\alpha \frac{R^{\prime}(w)}{R(w)-D-R(0)}=\frac{1-\alpha}{\rho-w}
$$

et ne dépend donc pas du salaire minimum. La solution de Nash prédit donc un point d'accumulation dans la distribution des salaires au niveau du salaire minimum et une diffusion nulle des hausses de salaire minimum au-delà. Ces deux propriétés paraissent contredire l'observation empirique. Comme le montre la Figure 1, la distribution des salaires observée en France ne présente pas de point d'accumulation, au-delà des erreurs d'arrondi. Par ailleurs, on retient généralement l'idée que les hausses de salaire minimum se diffusent dans la hiérarchie des salaires, jusqu'à environ 1,5 fois le SMIC (voir par exemple CSERC (1999)).

Le point d'accumulation au salaire minimum et l'absence de diffusion sur la distribution des salaires sont liés à l'axiome d'indépendance des choix non pertinents qui sous-tend la solution de Nash. Cet axiome implique en particulier que si le salaire négocié est supérieur au salaire minimum, alors une hausse marginale du salaire minimum n'a aucun effet sur le salaire négocié. Il semble donc qu'il faille renoncer à cet axiome pour serrer au plus près l'observation. Nous nous intéresserons dans cet article à un concept de solution qui ne présente pas ces inconvénients : la solution de Kalai-Smorodinsky (1975). Celle-ci remplace l'axiome d'indépendance des choix non pertinents par un axiome de monotonie individuelle qui exprime que si le gain maximal possible d'une des parties augmente, alors son surplus négocié doit également augmenter. Dans le problème de négociation salariale, le gain maximal de l'entreprise est obtenu quand le salaire négocié est égal au salaire minimum : il est égal à $(\rho-\underline{w})$. L'axiome de monotonie individuelle implique que le salaire négocié augmente avec le salaire minimum. La solution de Kalai-Smorodinsky induit donc une diffusion des hausses de salaire minimum. Formellement, cette solution est l'intersection du lieu des négociations efficaces avec le segment qui relie le point de menace $(R(0), 0)$ au point des gains 

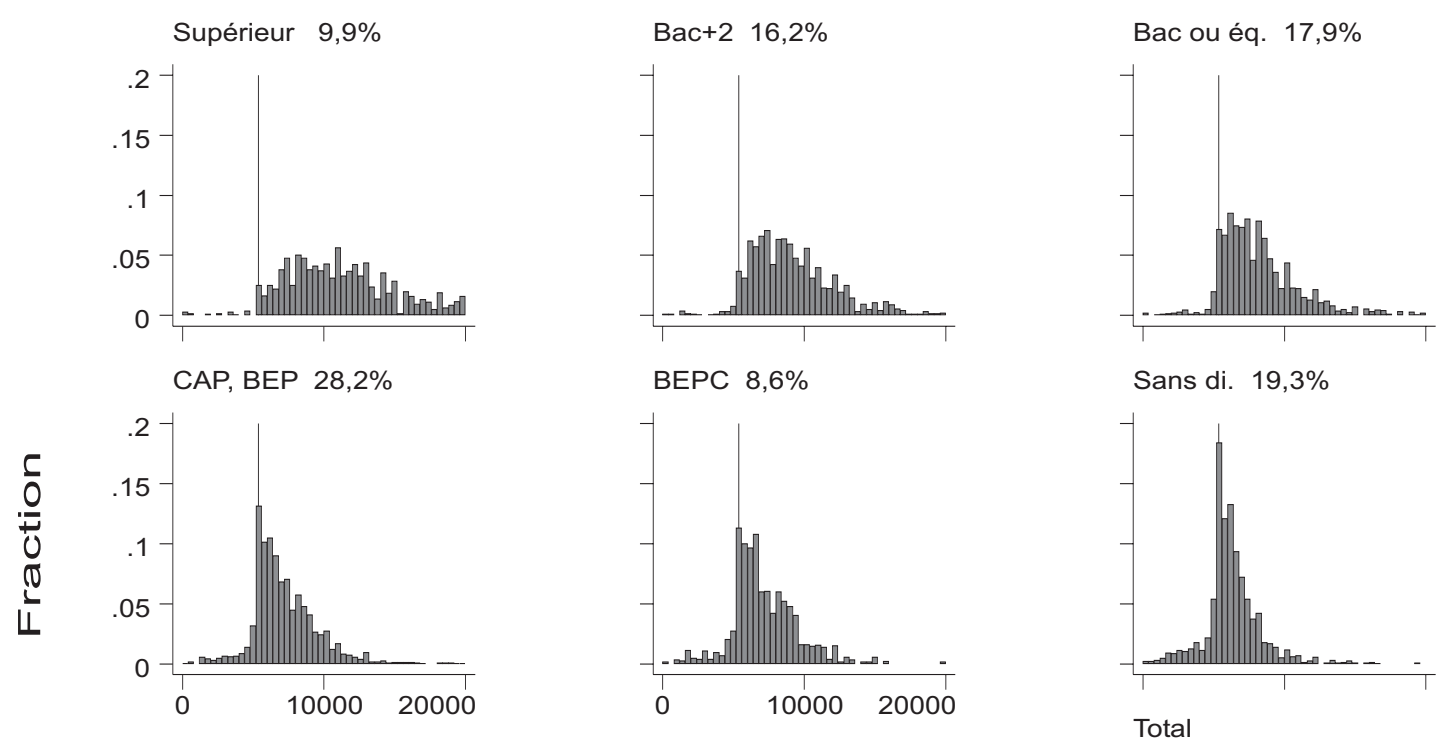

BEPC $8,6 \%$
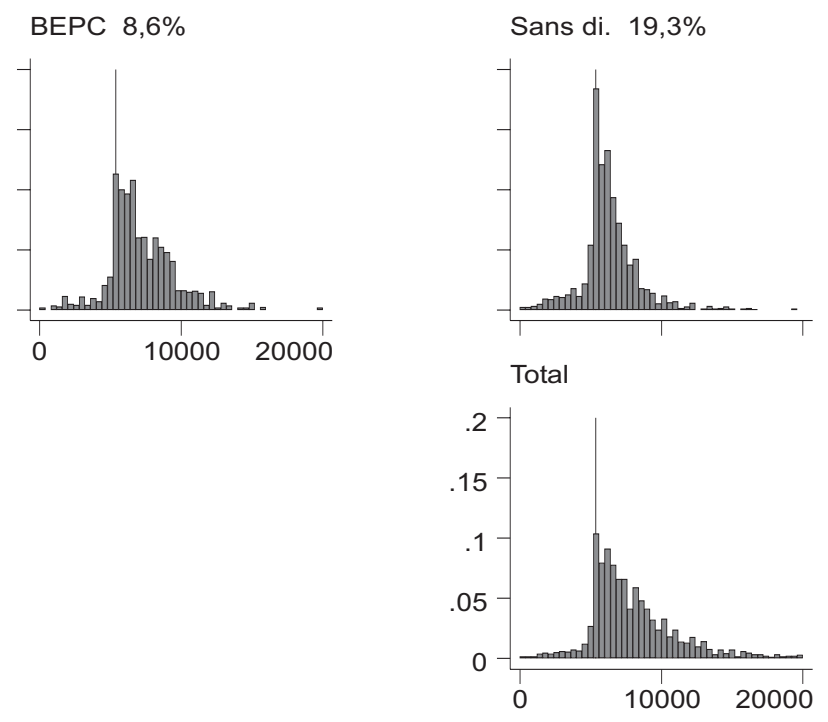

en équivalent temps plein

Distribution des salaires nets

FIG. 1: Les salaires dans l'échantillon 
maximaux $(R(\rho)-D, \rho-\underline{w})$. Des calculs élémentaires montrent que le salaire négocié est donné par

$$
\frac{R(w)-R(0)-D}{R(\rho)-R(0)-D}=\frac{\rho-w}{\rho-\underline{w}}
$$

Nous nous intéresserons en fait à la solution asymétrique ${ }^{1}$ qui assigne un pouvoir de négociation $0<\alpha<1$ au travailleur. Rappelons qu'il ne peut y avoir négociation salariale que si $\rho \geq \underline{w}$ et $R(\rho) \geq R(0)+D$. Formellement, le coût du travail est solution de l'équation :

$H(w, \rho, D, \underline{w})=(1-\alpha)(\rho-\underline{w})(R(w)-R(0)-D)-\alpha(\rho-w)(R(\rho)-R(0)-D)=0$.

Celle-ci s'écrit aussi

$$
\frac{1}{\alpha} \frac{R(w)-R(0)-D}{R(\rho)-R(0)-D}=\frac{1}{1-\alpha} \frac{\rho-w}{\rho-\underline{w}}
$$

Dans le domaine considéré, toute solution est telle que $(R(w)-R(0)-D)$ et $(\rho-w)$ sont de même signe. Nous supposons que la fonction $R$ est croissante. $H$ est alors strictement croissante en $w$ pour $\alpha>0$. Comme $H(\rho, \rho, D, \underline{w})$ est positif (strictement dès lors que $R(\rho)>R(0)+D$ et $\alpha<1$ ), on en déduit :

$$
w \leq \rho \quad \text { et } \quad R(w) \geq R(0)+D .
$$

Considérons maintenant

$$
H(\underline{w}, \rho, D, \underline{w})=(\rho-\underline{w})[(1-\alpha)(R(\underline{w})-R(0)-D)-\alpha(R(\rho)-R(0)-D)]
$$

Si $\alpha>1 / 2$, alors cette quantité est négative; et elle ne peut s'annuler que si $\rho=\underline{w}$. Le cas $\alpha<1 / 2$ conduit à des anomalies : il peut alors se faire (si $\rho$ est proche de $\underline{w})$ que $H(\underline{w}, \rho, D, \underline{w})$ soit positif, auquel cas $w$ n'est pas défini. Nous écarterons cette possibilité dans toute la suite de l'article.

On voit immédiatement que $H(w, \rho, D, \underline{w})$ décroît en $\underline{w}$. Par ailleurs, on calcule

$$
H_{\rho}^{\prime}=(1-\alpha)(R(w)-R(0)-D)-\alpha(\rho-w) R^{\prime}(\rho)-\alpha(R(\rho)-R(0)-D)
$$

soit en un point où $H=0$ :

$$
H_{\rho}^{\prime}=-\alpha(\rho-w) R^{\prime}(\rho)-\alpha(R(\rho)-R(0)-D) \frac{w-\underline{w}}{\rho-\underline{w}}<0
$$

De même,

$$
H_{D}^{\prime}=-(1-\alpha)(\rho-\underline{w})+\alpha(\rho-w)
$$

qui vaut

$$
H_{D}^{\prime}=-(1-\alpha)(\rho-\underline{w}) \frac{R(\rho)-R(w)}{R(w)-R(0)-D}<0
$$

en un point où $H=0$.

En résumé :

\footnotetext{
${ }^{1}$ Voir par exemple Thomson (1994).
} 
Lemme 1 Supposons $\alpha>1 / 2$ et la fonction $R(w)$ non décroissante. Alors pour les valeurs de $(\rho, D)$ qui vérifient

$$
\rho \geq \underline{w} \quad \text { et } \quad R(\rho) \geq R(0)+D
$$

la négociation salariale a un résultat unique $w$, solution de (1), tel que

$$
w \leq \rho \quad \text { et } \quad R(w) \geq R(0)+D .
$$

Cette solution $w$ est une fonction croissante du triplet $(\rho, D, \underline{w})$. De plus,

$$
\rho=\underline{w} \Leftrightarrow w=\underline{w}
$$

Par construction, le salaire négocié dépend du salaire minimum mais ne coïncide jamais avec celui-ci, sauf dans le cas de mesure nulle où $\rho=\underline{w}$. Il n'y a pas de point d'accumulation des salaires au salaire minimum. Les propriétés qualitatives de la solution de Kalai-Smorodinsky ne contredisent pas les deux faits stylisés mentionnés plus haut, qui nous ont fait écarter la solution de Nash.

On remarquera que le salaire négocié croît en la désutilité du travail, Ainsi et toutes choses égales par ailleurs, la présence d'enfants doit augmenter le salaire négocié. Ce résultat fait abstraction de phénomènes susceptibles d'apparaître dans des modèles de recherche d'emploi. Si par exemple les femmes sans enfant ont des coûts de recherche d'emploi plus faibles que les femmes qui ont des enfants, il est possible qu'elles obtiennent un salaire plus élevé, alors même qu'elles ont une désutilité du travail plus faible.

Nous avons supposé que la fonction $R$ était strictement croissante et continûment différentiable. Il est facile de voir que le Lemme 1 reste valable si $R$ reste strictement croissante mais n'est que continue. En revanche, la croissance de $R$ sur l'intervalle $[\underline{w}, \rho]$ est essentielle à nos résultats. Nous devrons donc éliminer plus loin les rares observations pour lesquelles $R$ n'est pas croissante.

\section{Spécification économétrique et méthode d'esti- mation}

La spécification que nous retenons ici s'appuie sur nos travaux antérieurs (Laroque et Salanié (2000, 2001a, 2001b)). La décision de participation est liée au revenu tiré de l'activité. Le salaire minimum exclut de l'emploi les personnes trop peu qualifiées. Enfin, même pour les femmes très qualifiées qui souhaitent travailler, il y a une probabilité de se trouver en non emploi frictionnel ou conjoncturel, ce que nous appelons autre non emploi. L'innovation de cet article tient uniquement à la détermination du salaire, qui ne résulte plus de l'égalité du coût du travail à la productivité comme précédemment, mais d'une négociation entre l'entreprise et l'employée selon les termes décrits dans la section précédente. 
La productivité est représentée par l'équation linéaire

$$
\ln \rho=X a+\sigma \varepsilon
$$

où les variables explicatives $X$ comprennent l'âge de fin d'études, son carré, l'expérience (mesurée, faute de mieux, par la différence entre l'âge au moment de l'enquête et l'âge de fin d'études) et son carré, et des indicatrices de diplôme. La productivité n'est pas observée directement. Elle détermine, conjointement avec la désutilité du travail et le revenu d'inactivité, le coût du travail des employées lors de la négociation, par la formule (1). C'est par ce biais que nous pourrons estimer les paramètres $a$ et $\sigma$. Notons que notre mise en œuvre de la solution de Kalai-Smorodinsky suppose que le salaire minimum est une barrière infranchissable : il est illégal de rémunérer une employée au-dessous du (coût du) salaire minimum, et cette loi est parfaitement respectée. Dans ces conditions, une femme qui souhaite travailler et dont la productivité $\rho$ est inférieure au coût du salaire minimum à temps plein $\underline{w}$ ne pourra pas trouver un emploi.

Nous dérivons la décision de participation d'un modèle à choix multiple où l'individu est supposé comparer les équivalents monétaires suivants des utilités de deux états ${ }^{2}$ :

$$
\begin{cases}R(w)-d+\frac{\eta_{1}}{\gamma} & \text { (travail à temps plein) } \\ R(0)+r \varepsilon+\frac{\eta_{0}}{\gamma} & \text { (non-participation) }\end{cases}
$$

Le premier terme de chacune des formules fait intervenir la fonction $R(w)$, qui donne les ressources financières nettes du ménage quand la femme travaille à un coût $w$ pour l'employeur, $w=0$ quand elle ne travaille pas. Cette fonction dépend d'arguments implicites dont les plus importants sont le salaire éventuel du conjoint et la composition familiale du ménage; elle résulte de l'application mécanique des barèmes et ne dépend d'aucun paramètre à estimer.

Le terme $d$ représente les facteurs observables par l'économètre de la désutilité du travail. On remarquera que selon les termes du modèle théorique,

$$
D=d+r \varepsilon+\frac{\eta_{0}-\eta_{1}}{\gamma}
$$

On pose :

$$
d=Z \beta=R(0) Z_{r} \beta_{r}+Z_{c} \beta_{c},
$$

où $Z_{r}$ et $Z_{c}$ sont des vecteurs de variables explicatives qui comprennent l'âge de la femme, son statut marital, le nombre et l'âge des enfants du ménage. La présence de $R(0)$ dans ces désutilités mérite commentaire. D'une part, les équations de participation habituellement estimées prennent en compte un effet revenu qui passe par les revenus non-salariaux de l'individu considéré, ce qui correspond

\footnotetext{
${ }^{2}$ Nous écartons de cette étude les femmes qui travaillent à temps partiel (Laroque et Salanié (2001b) analysent un modèle à trois états, en rajoutant le travail à mi-temps).
} 
exactement à $R(0)$ dans nos notations. Par ailleurs, la désutilité du travail dépend en partie des coûts de garde des enfants, qui varient habituellement avec le niveau de revenus du ménage, dont $R(0)$ est un indicateur simple.

Enfin, les facteurs stochastiques inobservables du choix de participation sont représentés par les variables $\eta$ qui sont supposées indépendamment et identiquement distribuées selon une loi à valeurs extrêmes, si bien que la probabilité de participation, du point de vue de l'économètre ${ }^{3}$, est donnée par l'expression logit habituelle :

où

$$
p=\frac{a_{e}}{a_{e}+a_{n e}}
$$

$$
\left\{\begin{array}{l}
a_{e}=\exp (\gamma(R(w)-d)) \\
a_{n e}=\exp (\gamma(R(0)+r \varepsilon)) .
\end{array}\right.
$$

Le terme $r \varepsilon$ autorise une corrélation entre l'équation de productivité et le choix de participation. Si $r$ est par exemple positif, les femmes qui ont une productivité inhabituellement élevée au regard de leurs caractéristiques observées sont aussi celles qui sont le plus réticentes à se porter sur le marché du travail, toutes choses égales par ailleurs.

A ce stade, une femme ne peut trouver un emploi que si elle le souhaite (compte tenu de l'accroissement de ressources que son salaire procurera à son ménage) et si elle est suffisamment productive pour que les employeurs lui offrent un salaire supérieur au salaire minimum. Toutefois, il existe certainement des femmes qui souhaitent travailler, sont suffisamment productives pour gagner le salaire minimum, mais ne parviennent pas à obtenir un emploi. Il se peut par exemple que ces femmes soient en transition entre deux emplois (chômage frictionnel), ou qu'elles soient victimes d'une conjoncture défavorable (chômage keynésien). L'idéal serait bien évidemment de pouvoir modéliser ces deux causes de non-emploi (et éventuellement d'autres) de manière structurelle. Nous nous limitons dans cette étude à postuler l'existence d'une probabilité de trouver un emploi conditionnelle à ce que l'on en cherche un et que l'on soit suffisamment productive pour être susceptible d'en obtenir un. Ainsi, nous notons $P$ la probabilité ${ }^{4}$ qu'une femme qui souhaite travailler et dont la productivité $\rho$ est supérieure au coût du salaire minimum obtienne effectivement un emploi.

Compte tenu de l'hétérogénéité des situations résumées dans l'autre nonemploi, nous nous sommes contentés d'autoriser la probabilité $P$ à dépendre de l'âge et du diplôme $j$ des femmes concernées. Après plusieurs expérimentations, nous avons finalement retenu la forme suivante :

$$
P=\exp \left(-\delta_{j}^{2}-\delta^{2}(\text { âge }-25)\right) .
$$

\footnotetext{
${ }^{3}$ La femme qui prend la décision connaît les valeurs prises par les variables $\eta$, et choisit la perspective qui lui donne la plus grande utilité, en équivalent monétaire.

${ }^{4} \mathrm{Il}$ ne faut pas confondre $p$, qui désigne la probabilité de participation du seul point de vue de l'économètre, et ce $P$ qui décrit l'action d'un aléa tant du point de vue de la personne considérée que de l'économètre.
} 
Cette forme fonctionnelle est clairement ad hoc, mais elle ajuste mieux les données que d'autres que nous avons essayées - et elle a l'avantage évident de contraindre ces probabilités à évoluer entre 0 et 1 .

L'identification des diverses équations du modèle de négociation présente des similitudes avec celle du modèle concurrentiel, mais aussi une différence importante. Comme dans le modèle concurrentiel (voir Laroque et Salanié (2001a, 2001b)), certaines équations sont identifiées non paramétriquement. Ainsi la forme de $P$ est observable dès lors qu'il existe des personnes qui désirent travailler et qui ont un salaire potentiel très élevé du fait de leur niveau d'études et de leur expérience : la seule raison qu'elles soient en non emploi est le facteur $P$. De même, la forme réduite de l'équation de salaire issue de la négociation est non paramétriquement identifiable au-dessus du salaire minimum (en dessous, l'identification repose sur les hypothèses faites sur la distribution des aléas). Dans le modèle concurrentiel l'identification séparée de la demande (équation de productivité) et de l'offre (équation de participation) venait de l'exclusion des diplômes de l'équation de participation et de l'exclusion des variables représentatives de la situation familiale (nombre d'enfants,..) de l'équation de productivité. Mais ici la négociation mélange tout : l'identification de la productivité et de la participation dépend crucialement de la forme précise de la solution de Kalai-Smorodinsky.

Le calcul de la vraisemblance diffère selon que la personne est ou non employée. Dans le premier cas, on observe le coût de son travail $w$, pas dans le second. L'élément essentiel du calcul est la densité jointe inconditionnelle du couple $(D, \rho)$ qui s'écrit :

$$
g(D, \rho)=\frac{1}{\sigma \rho} \phi\left(\frac{\ln \rho-X a}{\sigma}\right) \gamma L\left[\gamma\left\{D-Z \beta-r\left(\frac{\ln \rho-X a}{\sigma}\right)\right\}\right],
$$

où $\phi(x)=\exp \left(-x^{2} / 2\right) / \sqrt{2 \pi}$ et $L(x)=\exp (-x) /[1+\exp (-x)]^{2}$ sont les densités respectives de la loi normale centrée réduite et de la loi logistique. Intervient également l'expression de la probabilité $P$.

Considérons d'abord le cas d'une personne sans emploi. Il nous faut calculer la probabilité de ne pas être employée lorsqu'on ne connaît pas $w$. Pour ce faire, on calcule la probabilité inconditionnelle d'être employée, $P_{E}$, la probabilité cherchée étant le complément à un de cette grandeur. Sauf chômage conjoncturel ou frictionnel, on est employé dès lors que les deux conditions du lemme, $\rho \geq \underline{w}$ et $R(\rho) \geq R(0)+D$, sont vérifiées. Donc :

$$
P_{E}=P \int_{\underline{w}}^{\infty} d \rho \int_{-\infty}^{R(\rho)-R(0)} g(D, \rho) d D .
$$

La dernière intégrale a une expression analytique explicite (logistique), ce qui nous ramène à une intégrale simple :

$$
P_{E}=
$$




$$
P \int_{\underline{w}}^{\infty} \frac{1}{\sigma \rho} \phi\left(\frac{\ln \rho-X a}{\sigma}\right) \frac{1}{1+\exp \left[-\gamma\left\{R(\rho)-R(0)-Z \beta-r \frac{\ln \rho-X a}{\sigma}\right\}\right]} d \rho .
$$

Cette intégrale est calculée numériquement par une approximation de GaussLaguerre avec huit points. Rappelons que l'approximation de Gauss-Laguerre est donnée par

$$
\int_{0}^{\infty} f(\varepsilon) d \varepsilon \equiv \sum_{i=1}^{8} q_{i} e^{\varepsilon_{i}} f\left(\varepsilon_{i}\right)
$$

où les points $\varepsilon_{i}$ et les poids $q_{i}$ sont tabulés dans les ouvrages d'analyse numérique. Une application naïve de cette formule se heurte au fait que les $\varepsilon_{i}$ tabulés sont relativement petits par rapport à l'ordre de grandeur de $\rho$, si bien que l'échantillonnage résultant de l'intégrale est de mauvaise qualité. Pour appliquer la méthode de Gauss-Laguerre, nous choisissons donc huit valeurs pour $\rho$ de la forme $\rho_{i}=\underline{w} \exp \left(0,3 \varepsilon_{i}\right)$, où 0,3 est l'ordre de grandeur de l'écart type des équations de Mincer sur le logarithme du coût du travail et $\varepsilon_{i}$ les valeurs de la table de Gauss-Laguerre. L’intégrale est alors calculée par la formule :

$$
\int_{\underline{w}}^{\infty} f(\rho) d \rho \equiv \sum_{i=1}^{8} 0,3 q_{i} e^{\varepsilon_{i}} \rho_{i} f\left(\rho_{i}\right) .
$$

Analysons maintenant le cas d'une personne employée à un coût du travail $w$. Il nous faut cette fois calculer la probabilité d'être employée, $P_{E, w} d w$, avec un coût du travail compris entre $w$ et $(w+d w)$.

Dans le plan $(D, \rho)$, l'ensemble des couples qui peuvent conduire à un $w$ donné, $w \geq \underline{w}$, est une courbe dans une portion du plan limitée par $\rho \geq w$ et $D \leq(R(w)-R(0))$, courbe définie implicitement par l'équation $H(w, \rho, D, \underline{w})=$ 0 . Cette équation donne une expression explicite de $D$ en fonction de $\rho$ et $w$, croissante en $w$, dès lors que $\alpha$ est différent de 1 . Pour $\alpha=1$, on retombe sur le modèle concurrentiel où le coût du travail est égal à la productivité, $\rho=w$ : la courbe n'est plus paramétrable en $\rho$; on ne peut calculer $D$ en fonction de $\rho$. Comme le cas $\alpha=1$ est important, il nous faut une méthode de calcul robuste au voisinage de cette valeur. Nous avons donc choisi de résoudre numériquement l'équation implicite en $\rho$, en paramétrant en $D$. Soit $\rho(w, D)$ la solution, définie pour $D \leq R(w)-R(0)$. A $D$ fixé, la probabilité que le coût du travail soit compris entre $w$ et $w+d w$ est, d'après la formule de changement de variable ${ }^{5}$ :

$$
g(D, \rho(w, D)) \frac{\partial \rho}{\partial w}(w, D) d w
$$

ce qui donne l'expression :

$$
P_{E, w}=P \int_{-\infty}^{R(w)-R(0)} g(D, \rho(w, D)) \frac{\partial \rho}{\partial w}(w, D) d D .
$$

\footnotetext{
${ }^{5}$ La fonction $\rho(w, D)$ est strictement croissante en $w$ pour $\alpha$ supérieur à $1 / 2$, comme le montre la formule de la dérivée partielle plus bas.
} 
Aux points de différentiabilité de $R(w)$, le théorème des fonctions implicites donne :

$$
\frac{\partial \rho}{\partial w}(w, D)=\frac{(1-\alpha)(\rho-\underline{w}) R^{\prime}(w)+\alpha[R(\rho)-R(0)-D]}{\alpha(\rho-w) R^{\prime}(\rho)+\alpha[R(\rho)-R(0)-D]-(1-\alpha)[R(w)-R(0)-D]},
$$

où la valeur de $\rho$ au second membre est égale à $\rho(w, D)$. Le calcul pratique est plus compliqué que dans le cas des non employées. Là aussi, on approxime l'intégrale par la formule de Gauss-Laguerre avec huit points en renormalisant les $\varepsilon_{i}$ tabulés. Les valeurs choisies pour $D$ sont de la forme $D_{i}=R(w)-R(0)-\varepsilon_{i} / 0,0004$, où 0,0004 est voisin des valeurs estimées de $\gamma$ dans nos travaux antérieurs. La difficulté est que l'on n'a pas d'expression explicite pour $\rho\left(w, D_{i}\right)$, solution en $\rho$ de $H\left(w, \rho, D_{i}, \underline{w}\right)=0$. Cette équation ne dépend des paramètres à estimer qu'à travers $\alpha$. Pour réduire le temps de calcul, on la résout une fois pour toutes sur une grille de dix valeurs de $\alpha$ entre 0,51 et 1 . La valeur de $\rho\left(w, D_{i}\right)$ au cours des itérations est calculée par interpolation sur cette grille de dix valeurs. La fonction $R$ n'est pas différentiable, et présente même des points de discontinuité ; la valeur de $R^{\prime}(\rho)$, qui rentre dans la formule de $\partial \rho / \partial w$ est prise égale à $[R(\rho+100)-$ $R(\rho)] / 100$. Finalement, on a :

$$
\int_{-\infty}^{R(w)-R(0)} g(D, \rho(w, D)) \frac{\partial \rho}{\partial w}(w, D) d D \equiv \sum_{i=1}^{8} \frac{q_{i}}{0,0004} e^{\varepsilon_{i}} g\left(D_{i}, \rho\left(w, D_{i}\right)\right) \frac{\partial \rho}{\partial w}\left(w, D_{i}\right)
$$

\section{Données et résultats d'estimation}

L'estimation porte sur un échantillon de femmes âgées de 25 à 49 ans, constitué à partir de l'enquête Emploi de janvier 1999. L'enquête ne dit rien sur les pensions de retraites, les préretraites et les revenus non salariaux. Ce manque d'information délimite les contours de notre étude. Nous excluons ainsi les ménages dont un des membres a plus de 55 ans (dont une proportion non négligeable est en préretraite) et ceux dont un des membres est travailleur indépendant ou employeur (l'enquête ne donne que les revenus salariaux). Nous avons également exclu les fonctionnaires, que leurs modalités de recrutement et leurs carrières placent dans un monde un peu à part et dont la détermination des salaires ne passe pas par une négociation individuelle.

Nous écartons les salariées employées qui déclarent travailler à temps partiel, ou qui déclarent une durée de travail hebdomadaire inférieure à trente heures ou supérieure à cinquante. Nous laissons de côté le petit nombre d'employées qui déclarent un salaire horaire inférieur au salaire minimum. Enfin nous ne retenons pas six observations de femmes employées dont le revenu au travail $R(w)$ est inférieur au revenu que leur prédit notre modèle du système socio fiscal $R(0)$ si elles restaient inactives : ces observations contredisent directement l'hypothèse de croissance de la fonction $R$, qui est essentielle pour le calcul de la vraisem- 
TAB. 1: Distribution de l'échantillon par diplômes

\begin{tabular}{|l|c|}
\hline Diplôme supérieur & 0,085 \\
Baccalauréat +2 ans & 0,124 \\
Baccalauréat, brevet prof. ou équiv. & 0,150 \\
CAP, BEP ou équivalent & 0,269 \\
BEPC seul & 0,084 \\
Aucun diplôme ou CEP & 0,287 \\
\hline
\end{tabular}

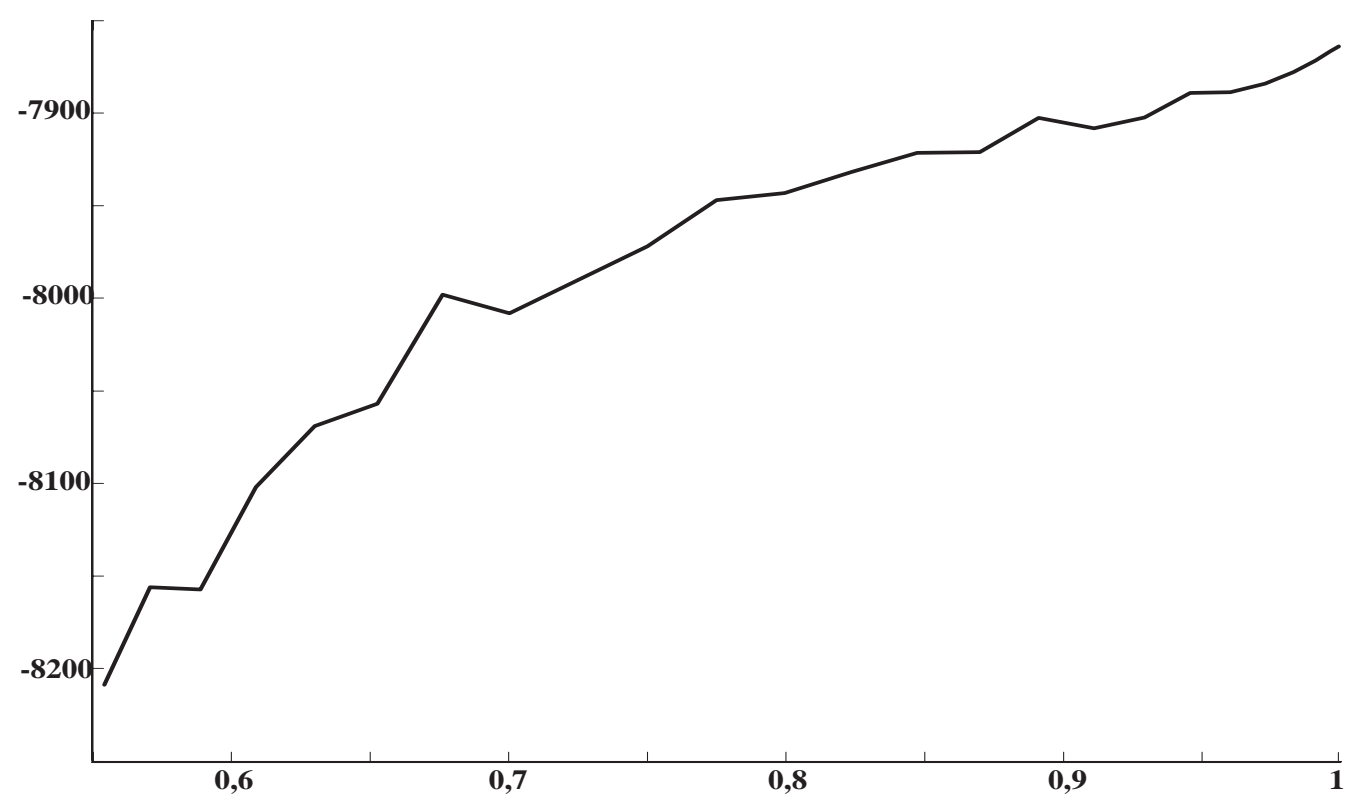

FIG. 2: Variation de la log-vraisemblance en fonction de $\alpha$

blance. Le traitement approprié de ces cas nous a semblé trop lourd en regard des bénéfices à en attendre.

En définitive, nous travaillons sur 13831 observations représentant 4,5 millions de femmes, se décomposant en 10573 femmes en couple et 3258 isolées, 6734 employées et 7097 sans emploi. Le salaire net mensuel des employées de l'échantillon est de 8600 francs. Lorsqu'elles sont en couple, leur conjoint travaille dans 88,3\% des cas. La distribution des diplômes des femmes de l'échantillon figure dans le tableau 1. Les femmes que nous étudions sont moins diplômées que l'ensemble de la population française, puisque nous avons écarté les fonctionnaires de l'Éducation Nationale. Près de 30\% n'ont pas de diplôme.

La maximisation de la vraisemblance sur les quarante-trois paramètres à estimer prend une dizaine d'heures sur un microordinateur muni d'un processeur 
TAB. 2: Équation de productivité

\begin{tabular}{|l|c|c||c|c|}
\hline \multirow{2}{*}{ Variable } & \multicolumn{2}{|c||}{$\alpha=1$} & \multicolumn{2}{c|}{$\alpha=0,8$} \\
& Coefficient & Écart-type & Coefficient & Écart-type \\
\hline Age de fin d'études & 0,1278 & 0,0111 & 0,1556 & 0,0125 \\
Age de fin d'études au carré & $-0,0024$ & 0,0003 & $-0,0029$ & 0,0003 \\
Expérience & 0,0446 & 0,0021 & 0,0474 & 0,0023 \\
Expérience au carré & $-0,0006$ & 0,0001 & $-0,0006$ & 0,0001 \\
Diplôme supérieur & 0,7326 & 0,0232 & 0,8058 & 0,0247 \\
Baccalauréat + 2 ans & 0,4880 & 0,0201 & 0,5256 & 0,0217 \\
Baccalauréat, brevet prof. ou équiv. & 0,3113 & 0,0176 & 0,3264 & 0,0194 \\
CAP, BEP ou équiv & 0,1619 & 0,0150 & 0,1671 & 0,0164 \\
BEPC seul & 0,1385 & 0,0187 & 0,1448 & 0,0207 \\
Constante & 7,0880 & 0,1157 & 6,7881 & 0,1327 \\
Écart type & 0,3165 & 0,0045 & 0,3456 & 0,0030 \\
\hline
\end{tabular}

TAB. 3: Équation de participation

\begin{tabular}{|l|c|c||c|c|}
\hline \multirow{2}{*}{ Variable } & \multicolumn{2}{|c||}{$\alpha=1$} & \multicolumn{2}{c|}{$\alpha=0,8$} \\
& Coefficient & Écart-type & Coefficient & Écart-type \\
\hline$R(0)$ & 0,190 & 0,063 & 0,260 & 0,078 \\
$R(0) \times$ enfants de moins de 3 ans & $-0,155$ & 0,030 & $-0,201$ & 0,038 \\
$R(0) \times$ enfants entre 3 et 6 ans & $-0,148$ & 0,017 & $-0,207$ & 0,022 \\
$R(0) \times$ enfants entre 6 et 18 ans & 0,035 & 0,018 & 0,019 & 0,021 \\
$R(0) \times$ indicatrice mariée civilement & 0,064 & 0,062 & 0,124 & 0,077 \\
en couple & -5127 & 909 & -6068 & 1138 \\
isolée & -5815 & 770 & -6692 & 972 \\
nb enfants de moins de 3 ans & 5042 & 556 & 6435 & 742 \\
nb enfants entre 3 et 6 ans & 4442 & 360 & 5720 & 494 \\
nb enfants entre 6 et 18 ans & 1779 & 279 & 2519 & 372 \\
âge & 122 & 26 & 47 & 31 \\
indicatrice mariée civilement & 22 & 690 & -884 & 875 \\
$r$ & 2151 & 191 & 1833 & 211 \\
$1000 \gamma$ & 0,435 & 0,028 & 0,371 & 0,025 \\
\hline
\end{tabular}


Pentium 4 cadencé à 1,5 GHz. La vraisemblance a plusieurs maxima locaux. La figure 2 présente la valeur du maximum de la log-vraisemblance maximisée sur les quarante-deux coefficients restants quand on fait varier $\alpha$ entre 0,51 et 1 (il ne semble pas qu'il y ait de maxima multiples à $\alpha$ fixé). Le maximum global se trouve en $\alpha=1$ : sur notre échantillon, le modèle de négociation salariale individuelle est rejeté au profit du modèle concurrentiel. Il y a au moins quatre autres maxima locaux, pour $\alpha$ voisin de $0,67,0,77,0,89$ et 0,946 . La courbe est régulière, de sorte que la différence entre le dernier maximum local et le maximum global n'est pas très importante : pour $\alpha=0,946$, la log-vraisemblance est égale à -7889 , alors qu'elle vaut -7863 en $\alpha=1$. Il n'est pas exclu qu'une spécification un peu différente conduise à faire jouer un rôle à la négociation salariale. Nous ne commenterons pas en détail les résultats associés au maximum global : le modèle concurrentiel a fait l'objet de plusieurs travaux antérieurs (Laroque et Salanié $(2000,2001 \mathrm{a}, 2001 \mathrm{~b}))$. On cherchera plutôt à illustrer les effets potentiels de la négociation salariale en comparant le comportement du modèle lorsque $\alpha$ est égal à 1 avec $\alpha$ égal à 0,9 ou 0,8 . Pour $\alpha$ égal à 0,8 , la log-vraisemblance vaut -7943 : elle est inférieure de 80 points au maximum global.

Les estimations des coefficients des équations de productivité et de participation dans les deux cas $\alpha=1$ et 0,8 sont fournies dans les tableaux 2 et 3 respectivement. Pour la plupart des coefficients, la différence entre les deux estimations est inférieur au double de l'écart type. Pour $\alpha=0,8$, la précision du modèle, mesurée par l'écart type de l'erreur $\sigma$ de l'équation de productivité et l'inverse de $\gamma$ pour la participation, est moins bonne que pour $\alpha=1$. Quand $\rho$ devient grand à $D$ et $\underline{w}$ fixé, on voit facilement que $w$ est asymptotiquement équivalent à $\alpha \rho$. Pour retrouver une forme réduite similaire pour l'équation de salaire lorsqu' $\alpha$ varie, on peut donc s'attendre à trouver que les coefficients des variables qui interviennent uniquement dans la détermination de la productivité décroissent en $\alpha$. C'est bien ce que fournit l'estimation.

\section{Quelques cas-type}

Comme nous l'avons expliqué plus haut, le coût du travail négocié $w$ est une fonction complexe de la productivité $\rho$, du coût du salaire minimum $\underline{w}$, du pouvoir de négociation des travailleurs $\alpha$ et des caractéristiques du ménage (à travers la fonction $R$ et la désutilité du travail $D$ ). Nous nous contenterons ici d'illustrer les variations de $w$ lorsque $\alpha=0,8$ en estimant la désutilité du travail par son espérance calculée à partir des estimateurs contraints en cette valeur de $\alpha$. Nous choisissons pour sujet une femme de 35 ans qui vit dans un HLM et qui peut être sans enfant, avoir un enfant de 2 ans, deux enfants de 2 et 5 ans, ou trois enfants de 2,4 et 5 ans.

Le tableau 4 évalue le coût du travail négocié $w$ pour trois niveaux de productivité en supposant que cette femme n'a pas de conjoint. Rappelons, pour 
TAB. 4: Femme seule : coût du travail négocié

\begin{tabular}{|c|r|r|r|r|}
\hline Nombre d'enfants / productivité & 0 & 1 & 2 & 3 \\
\hline 10000 & 9629 & 9656 & - & - \\
20000 & 17411 & 17798 & 19466 & - \\
30000 & 25350 & 25653 & 26446 & 28125 \\
\hline
\end{tabular}

TAB. 5: Mari sans emploi : coût du travail négocié

\begin{tabular}{|c|r|r|r|r|}
\hline Nombre d'enfants / productivité & 0 & 1 & 2 & 3 \\
\hline 10000 & 9632 & 9684 & - & - \\
20000 & 17487 & 17819 & - & - \\
30000 & 25455 & 25872 & 26546 & 28642 \\
\hline
\end{tabular}

interpréter ce tableau et les suivants, que le coût du salaire minimum était de 8505 francs par mois en 1999. Les niveaux de productivité choisis sont indiqués en ligne et le nombre d'enfants en colonne. Lorsqu'une case du tableau contient un tiret, c'est que dans la configuration correspondante, la femme étudiée ne souhaite pas travailler. Dans tous les cas, un enfant supplémentaire accroît le coût du travail négocié - quand la femme continue à travailler. Cet effet est très faible pour le premier enfant, mais il est assez important pour le deuxième et surtout le troisième enfant.

Le tableau 5 s'applique au cas d'une femme qui a un conjoint sans emploi. Il diffère très peu du tableau 4 , sauf pour le cas d'une femme de productivité égale à 20000 francs par mois et qui a deux enfants à charge; cette femme participerait si elle était seule, mais pas si elle était mariée. Enfin, le tableau 6 décrit la situation de cette femme quand son mari a un emploi qui lui rapporte un salaire net de 8000 francs par mois. Les modifications sont encore une fois relativement faibles, sauf pour la femme de productivité 20000 francs et à 2 enfants qui reprend un emploi, et pour une femme de productivité 30000 francs et avec 3 enfants à charge dont le coût du travail négocié est nettement plus faible que si son mari ne travaillait pas.

Les trois tableaux précédents font apparaître qu'en dehors bien sûr de la productivité, le nombre d'enfants à charge est le principal déterminant du coût du travail négocié. On peut également étudier la diffusion des mouvements du salaire minimum. Nous nous placerons pour ce faire dans le cas d'une femme seule sans enfant. Le tableau 7 donne son coût du travail négocié en fonction de sa 
TAB. 6: Mari employé : coût du travail négocié

\begin{tabular}{|c|r|r|r|r|}
\hline Nombre d'enfants / productivité & 0 & 1 & 2 & 3 \\
\hline 10000 & 9631 & 9640 & - & - \\
20000 & 17393 & 17570 & 18525 & - \\
30000 & 25306 & 25619 & 26364 & 27077 \\
\hline
\end{tabular}

TAB. 7: Effets de diffusion du salaire minimum

\begin{tabular}{|c|r|r|r|r|r|}
\hline$\underline{w} /$ productivité & 0,5 SMIC & 0,75 SMIC & 1 SMIC & 1,25 SMIC & 1,5 SMIC \\
\hline 10000 & 8612 & 9113 & 9629 & - & - \\
20000 & 16584 & 16989 & 17411 & 17848 & 18303 \\
30000 & 24578 & 24959 & 25350 & 25752 & 26165 \\
\hline
\end{tabular}

productivité et d'un salaire minimum qui varie entre 0,5 et 1,5 fois son niveau en 1999. Lorsque cette femme est peu productive, elle ne peut plus trouver d'emploi si le salaire minimum s'accroît trop. Pour des productivités supérieures, les effets de diffusion du salaire minimum sont non-négligeables. Ainsi, une hausse de $25 \%$ du salaire minimum en 1999 (soit 2100 francs par mois) aurait augmenté le coût du travail négocié de 400 francs par mois environ pour les femmes qui auraient conservé leur emploi.

\section{Simulations}

L'estimateur du maximum de vraisemblance suggère que tout le pouvoir de négociation revient aux travailleurs $(\alpha=1)$, si bien que le coût du travail négocié coïncide avec sa productivité. Dans ces conditions, une hausse du salaire minimum a des effets simples : elle réduit l'emploi sans augmenter les salaires et n'a donc que des effets négatifs sur la masse salariale. Quand $\alpha<1$ en revanche, une hausse du salaire minimum peut s'avérer bénéfique pour les travailleurs pris dans leur ensemble si la hausse induite de leurs salaires négociés fait plus que compenser l'effet de la baisse de l'emploi. Il est donc intéressant d'étudier les effets du salaire minimum en fonction de $\alpha^{6}$. Pour ce faire, nous considérerons trois valeurs ${ }^{7}$ de $\alpha: 1,0,9$ et 0,8 . Pour chacune de ces valeurs, les simulations se fondent sur l'estimateur du maximum de vraisemblance contraint correspondant. Les chiffres sont calculés sur tout l'échantillon en tirant dix valeurs des aléas dans leur loi

\footnotetext{
${ }^{6}$ Flinn (2001) se livre à un exercice similaire sur données américaines en calculant l'espérance d'utilité actualisée d'un chômeur après avoir estimé la valeur de $\alpha$.

${ }^{7}$ Nous aurions pu explorer des valeurs plus basses, mais celles-ci semblent peu probables au vu des données.
} 


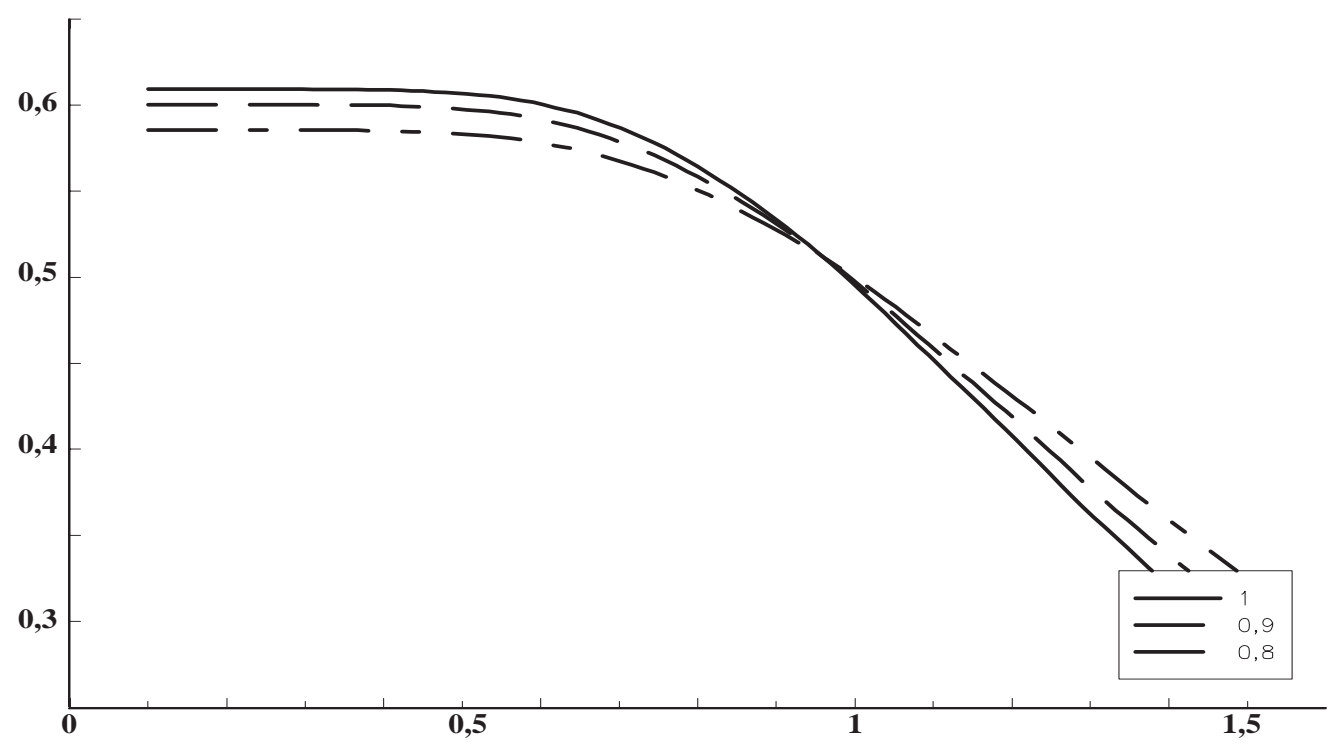

FIG. 3: Emploi et salaire minimum

et en affectant à chaque observation son poids de sondage. Nous ferons varier le coût du salaire minimum $\underline{w}$ entre 0,1 et 1,5 fois sa valeur en 1999 .

La figure 3 montre l'évolution de la probabilité d'emploi. Celle-ci vaut

$$
P_{E}=P \operatorname{Pr}(\rho>\underline{w} \text { et } R(\rho)>R(0)+D)
$$

et est donc toujours une fonction décroissante du salaire minimum ${ }^{8}$. Comme on le constate sur la figure, l'emploi décroît bien en fonction du salaire minimum, de manière très non-linéaire. Les effets négatifs du salaire minimum sur l'emploi deviennent sensibles au-delà de $70 \%$ du SMIC actuel environ.

La figure 4 illustre l'impact du salaire minimum sur la distribution des salaires nets quand $\alpha=0,8$. La courbe en trait plein montre la densité estimée de la distribution des salaires quand le salaire minimum est fixé à sa valeur observée en 1999; la courbe en pointillés représente la densité simulée si le salaire minimum augmente de $10 \%$. On constate qu'au-delà du déplacement du point de troncation de la distribution, la nouvelle distribution a un pic plus prononcé que la distribution observée. Les effets de la hausse du salaire minimum sur la distribution des salaires nets se font sentir jusqu'à environ 1, 8 fois le SMIC.

Lorsque le salaire minimum augmente, la distribution des salaires est tronquée plus haut et le salaire moyen des employées augmente donc lui aussi. Dans le

\footnotetext{
${ }^{8}$ En fait, la fonction $R$ dépend aussi de $\underline{w}$ à travers l'indexation des allégements de charges sur les bas salaires sur le salaire minimum. Cet effet est pris en compte dans les simulations.
} 


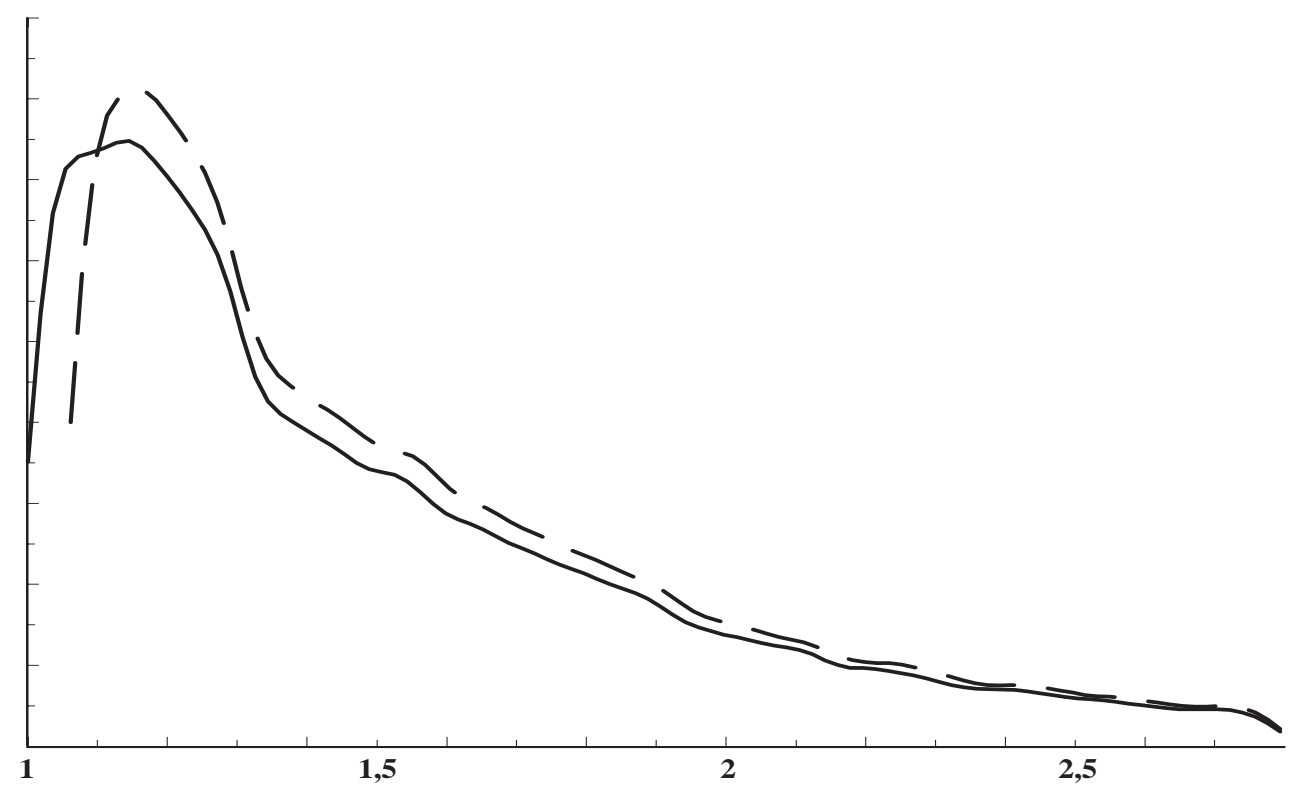

FIG. 4: Distributions simulées des salaires

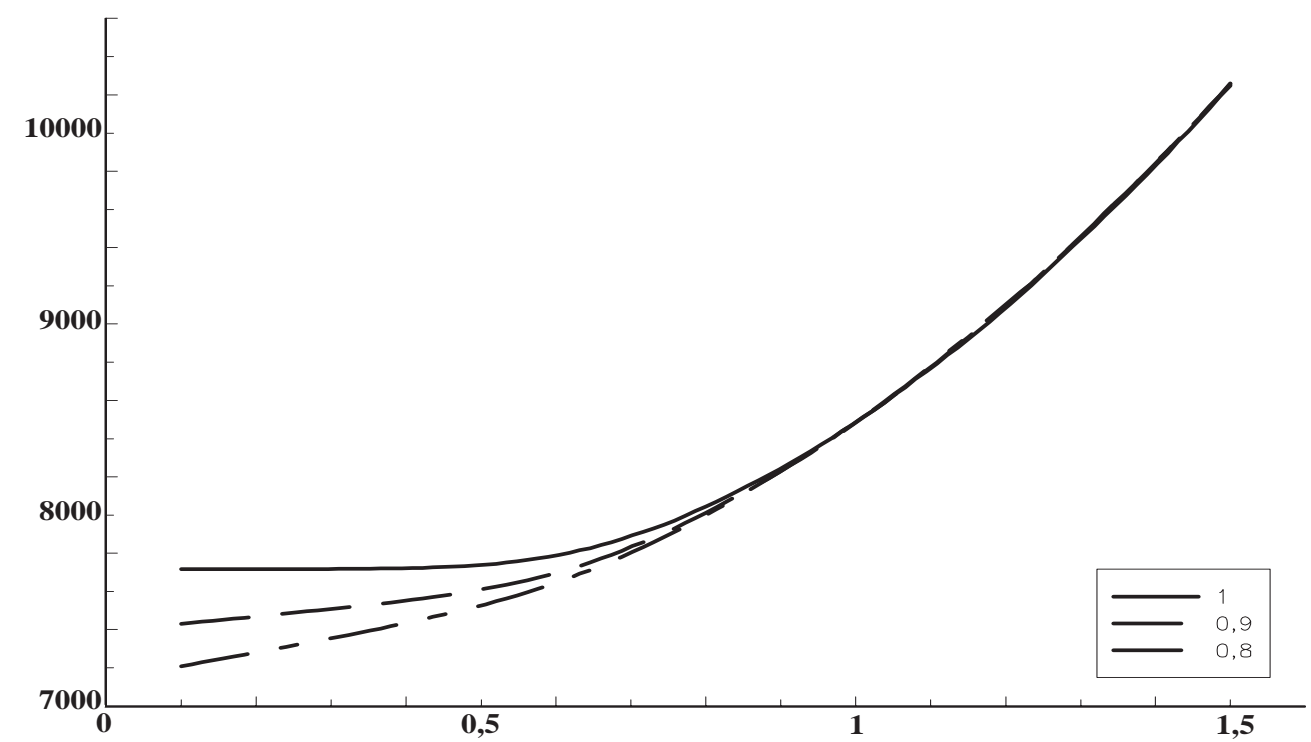

FIG. 5: Variation de la moyenne des salaires 


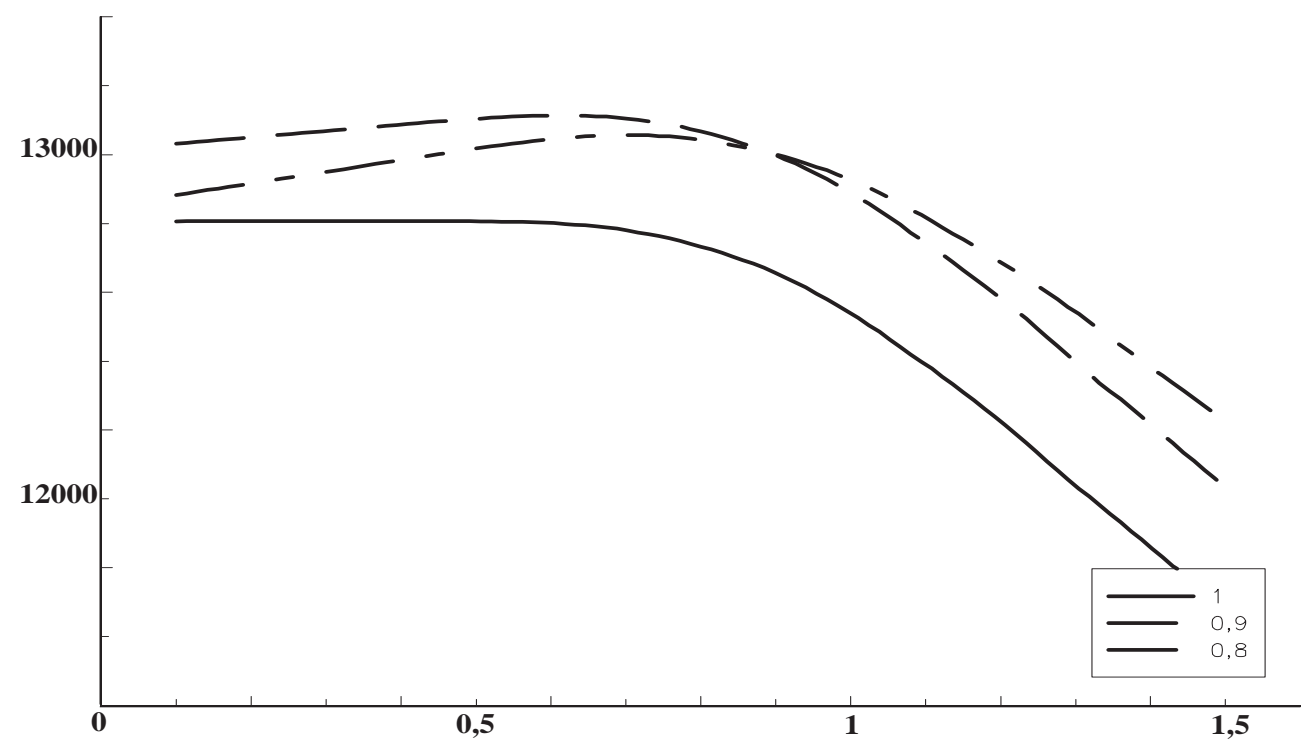

FIG. 6: Bien-être des ménages

modèle concurrentiel, le salaire individuel des personnes qui restent employées ne change pas. Quand $\alpha<1$, ce salaire croît avec $\underline{w}$. Ces deux effets du salaire minimum se traduisent par une croissance du salaire net moyen avec $\underline{w}$. La courbe qui décrit les variations de la moyenne des salaires des employées en fonction du salaire minimum est représentée sur la figure 5 pour les trois valeurs retenues de $\alpha$. Le premier effet (de troncation) domine pour des valeurs réalistes du salaire minimum. Le deuxième effet (lié au pouvoir de marché des entreprises) ne joue vraiment qu'en deçà de la moitié du SMIC actuel. Une hausse de $10 \%$ du SMIC en 1999 aurait augmenté le salaire net moyen d'environ 300 francs par mois, par le seul jeu de la troncation.

Les figures suivantes se rapportent au bien-être social. Soit $E$ la variable indicatrice de l'obtention d'un emploi, qui est donc une fonction décroissante de $\underline{w}$, et $N=1-E$. Alors le bien-être $B$ d'un ménage, en équivalent monétaire, est égal à

$$
B=(R(w)-D) E+R(0) N
$$

Le bien-être des femmes de l'échantillon est simplement obtenu en moyennant celui des différents ménages.

Comme $w$ est une fonction croissante de $\underline{w}$ si $\alpha<1$, et compte tenu du fait que $R(w)>R(0)+D$ par construction pour les employées, le sens de variation de $B$ est a priori ambigu. La figure 6 montre que quand $\alpha<1$, le bien-être de notre échantillon croît puis décroît; il atteint un maximum en $60 \%$ du SMIC actuel 


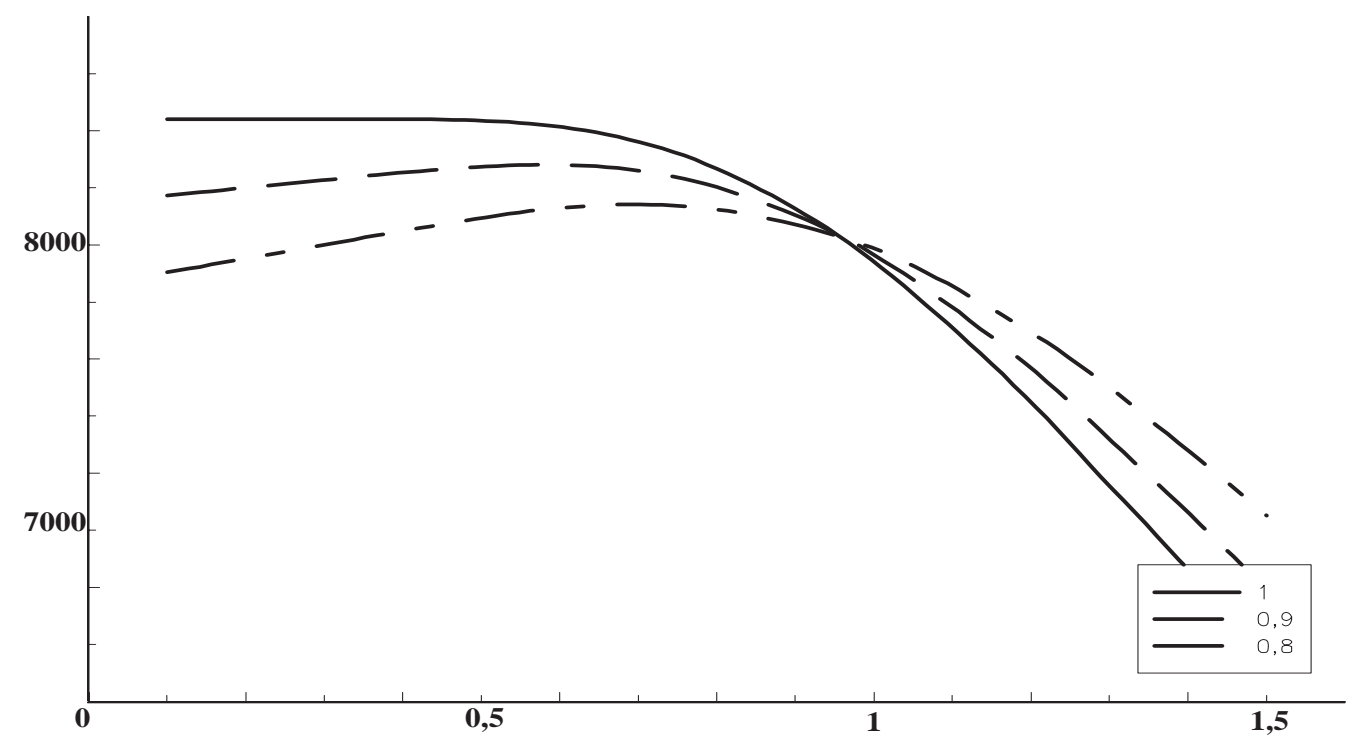

FiG. 7: Prélèvements nets sur les ménages

pour $\alpha=0,9$ et en $70 \%$ du SMIC actuel pour $\alpha=0,8$.

L'analyse de surplus ne se limite pas au bien-être des ménages; il faut de plus prendre en compte les variations des prélèvements qu'ils supportent. Soit donc $w_{c}$ le coût du travail du conjoint (pris égal à 0 si la femme est seule ou si son conjoint est sans emploi). Pour une femme employée, les prélèvements nets sur le ménage $^{9}$ sont égaux à $\left(w+w_{c}-R(w)\right)$; ils sont de $\left(w_{c}-R(0)\right)$ si la femme est sans emploi. Le prélèvement net payé par le ménage est donc

$$
T=\left(w+w_{c}-R(w)\right) E+\left(w_{c}-R(0)\right) N
$$

La figure 7 représente les variations de la somme des prélèvements $T$ en fonction du salaire minimum sur notre échantillon. On constate que $T$ décroît sur tout l'intervalle pour $\alpha=1$ et a un maximum en-deçà du SMIC actuel pour $\alpha=0,8$ et $\alpha=0,9$.

En additionnant $B$ et $T$, on obtient une mesure du bien-être des ménages qui prend en compte les variations du solde des prélèvements, dans la mesure où celui-ci peut être reversé aux ménages. On calcule immédiatement

$$
B+T=\left(w+w_{c}-D\right) E+w_{c} N
$$

\footnotetext{
${ }^{9}$ Il s'agit de l'ensemble des sommes transférées (ou versées, si le nombre est négatif) à la puissance publique. Pour une grosse part, c'est le ménage qui en est redevable, mais les cotisations sociales à la charge de l'employeur rentrent dans le champ.
} 


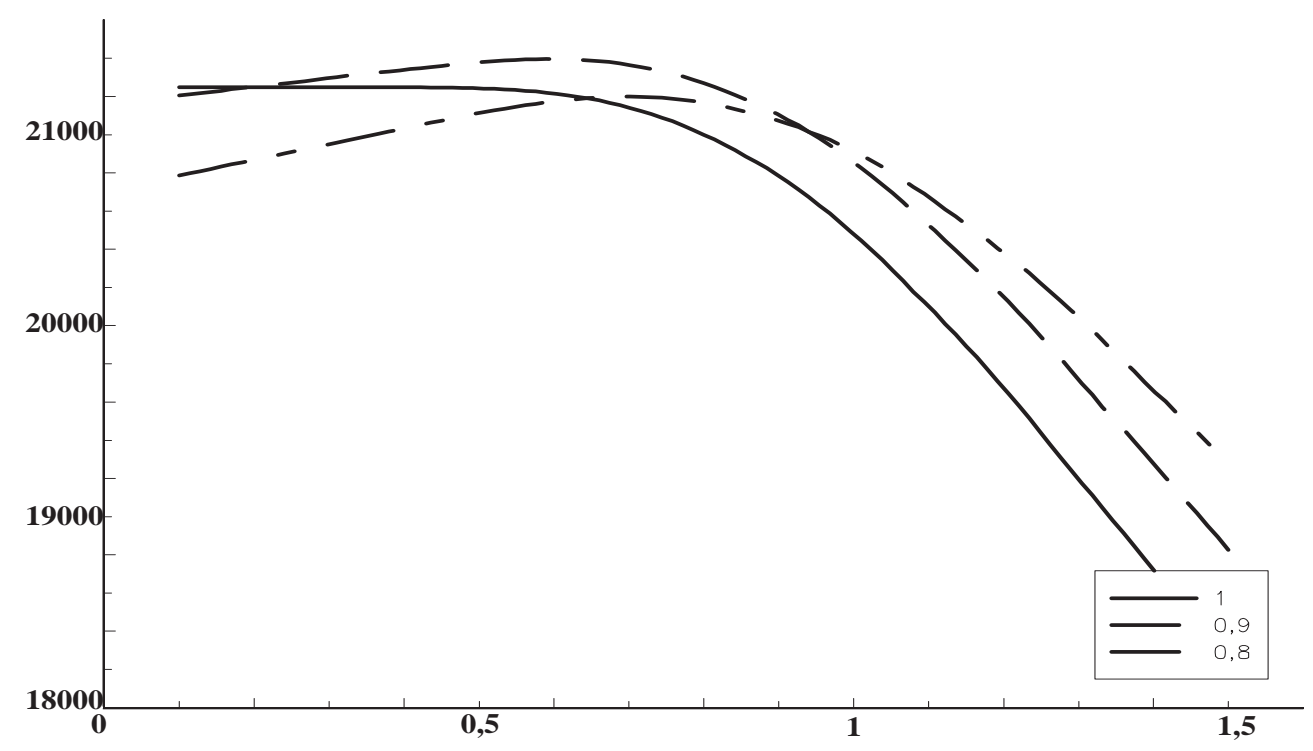

FIG. 8: Bien-être net des ménages

Là encore, le sens de variation de $(B+T)$ est ambigu. La figure 8 montre néanmoins que le bien-être net a un maximum unique proche de celui obtenu avec la première mesure du bien-être. Une hausse de $10 \%$ du SMIC au-delà du niveau observé réduirait le bien-être de 240 francs par mois et par personne (resp. 310, resp. 370) pour $\alpha=0,8$ (resp. 0, 9, resp. 1).

La mesure adoptée jusqu'ici néglige les profits des entreprises

$$
\pi=(\rho-w) E
$$

qui décroissent en $\underline{w}$ puisque $w \leq \rho, w$ est une fonction croissante et $E$ une fonction décroissante du salaire minimum. La figure 9 illustre la baisse des profits quand le salaire minimum augmente. Les profits sont bien sûr identiquement nuls pour $\alpha=1$, puisqu'alors $w=\rho$.

On peut justifier de négliger les profits si ceux-ci sont reversés à des actionnaires qui sont hors échantillon et dont le poids social est faible, du fait de leur richesse. L'hypothèse polaire consiste à affecter un poids égal aux revenus du travail et à ceux du capital. On obtient alors

$$
B+T+\pi=w_{c}+(\rho-D) E
$$

Lorsque $E=1$, par définition $R(\rho)>R(0)+D$. Comme le système socio-fiscal prélève une partie de la productivité des salariées, on a $R(\rho)-R(0)<\rho$ et donc 


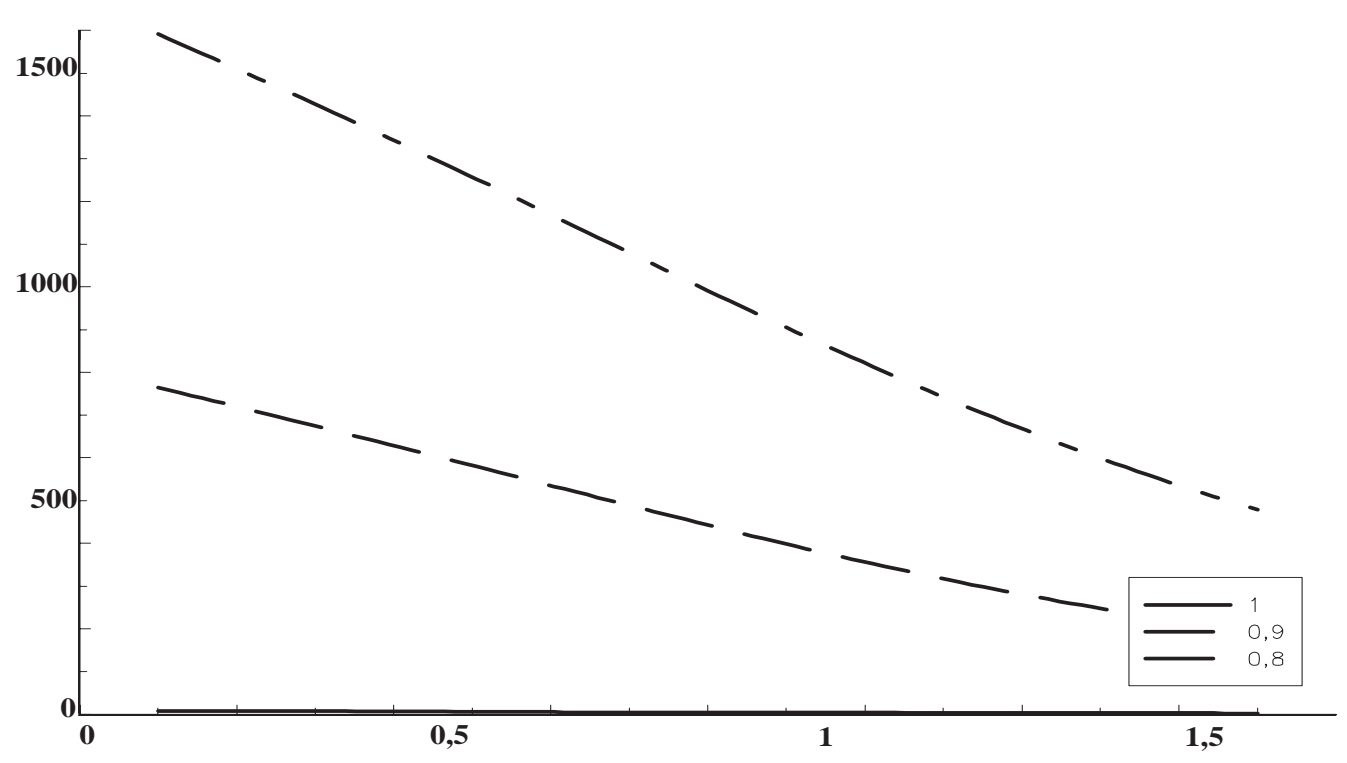

FIG. 9: Profit des entreprises

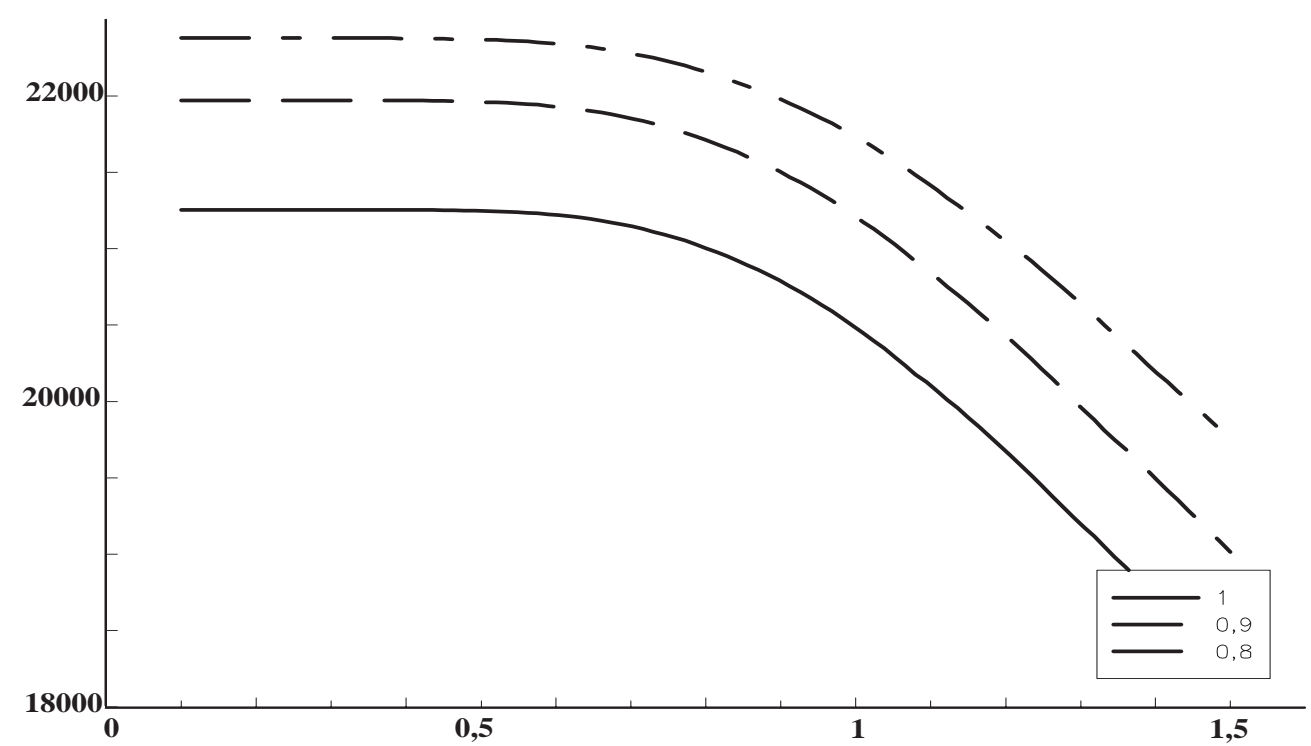

FIG. 10: Surplus social 
a fortiori $\rho>D$ si $E=1$. Le surplus social est donc une fonction décroissante du salaire minimum, comme l'indique la figure 10. La perte de surplus social induite par une hausse de 10\% du SMIC en 1999 aurait représenté 310 francs par mois et par personne (resp. 360, resp. 370) pour $\alpha=0,8$ (resp. 0,9, resp. 1).

\section{Conclusion}

Cette première tentative d'estimer un modèle de négociations salariales sur données individuelles conduit à un rejet au profit du modèle de formation concurrentielle des salaires. Pour être plus précis, les variables qui accroissent la désutilité du travail ou le taux de prélèvement socio-fiscal marginal ne semblent pas influencer le coût du travail observé. Cela n'est pas entièrement surprenant. Comme nous l'avons évoqué en introduction, les négociations salariales ne se placent souvent pas au niveau individuel et notre spécification préférée serait une négociation collective. Si par exemple la négociation collective se fondait sur la prise en considération d'un "individu moyen" d'un bassin d'emploi dans la solution de Kalai-Smorodinsky, alors la méthode appliquée dans cet article comporterait une erreur sur les variables. Dans un modèle linéaire (ce qui est très loin d'être notre cas), les estimateurs de $(1-\alpha)$ seraient alors affectés d'un biais vers zéro. Cet argument pourrait expliquer un estimateur de $\alpha$ très proche de un; mais nous obtenons en fait un estimateur égal à un. Ce résultat contredit les estimations obtenues dans la littérature dans un contexte complètement différent et rappelées en introduction. Ce sujet important nous paraît mériter des études plus approfondies.

\section{Bibliographie}

Abowd, J. et L. Allain (1996), "Compensation Structure and Product Competition", Annales d'économie et de statistique, 41-42, 207-217.

Abowd, J., F. Kramarz et D. Margolis (1999), "High Wage Workers and High Wage Firms", Econometrica, 67, 251-331.

Abowd, J. et T. Lemieux (1993), "The Effects of Product Market Competition on Collective Bargaining Agreements : The Case of Foreign Competition in Canada", Quarterly Journal of Economics, 108, 983-1014.

Blanchflower, D., A. Oswald et P. Sanfey (1996), "Wages, Profits, and Rent-sharing", Quarterly Journal of Economics, 111, 227-251.

Cahuc, P. et A. Zylberberg (2000), Le marché du travail, de Boeck.

CSERC (1999), Le SMIC : salaire minimum de croissance, La Documentation Française.

Eckstein, Z. et K. Wolpin (1995), "Duration to First Job and the Return to Schooling : Estimates from a Search Matching Model", Review of Economic 
Studies, 62, 263-286.

Flinn, C. (2001), "Minimum Wage Effects under Search with Bargaining", mimeo.

Gianella, C. (2002), Un réexamen des déterminants de l'emploi à partir de données microéconomiques, thèse de doctorat de Paris I.

Kalai, E. et M. Smorodinsky (1975), "Other Solutions to Nash's Bargaining Problem", Econometrica, 43, 513-518.

Kramarz, F. (2002), "Bargaining and International Trade", mimeo CREST.

Laroque G. et B. Salanié (2000), "Une décomposition du non emploi en France", Economie et Statistique, 331, 47-66.

Laroque G. et B. Salanié (2001a), "Labor Market Institutions and Employment in France", à paraître dans le Journal of Applied Econometrics.

Laroque G. et B. Salanié (2001b), Institutions et emploi : le marché du travail des femmes en France, mimeo, CREST.

Layard, R., S. Nickell et R. Jackman (1991), Unemployment, Oxford University Press.

Pissarides, C. (2000), Equilibrium Unemployment Theory, MIT Press.

Thomson, W. (1994), "Cooperative Models of Bargaining", in Handbook of Game Theory, vol. 2, R. Aumann et S. Hart eds.

van Reenen, J. (1996), "The Creation and Capture of Rents : Wages and Innovation in a Panel of UK Companies", Quarterly Journal of Economics, 111, 195-226. 